\title{
Increasing of horizontal velocity of particles leaving a belt conveyor
}

\author{
Abraão Tavares ${ }^{1, \star}$ and Allbens Faria ${ }^{1, \star \star}$ \\ ${ }^{1}$ Departamento de Física e Matemática, Centro Federal de Educação Tecnológica de Minas Gerais, Av. Amazonas, 7675, Belo \\ Horizonte, Brazil.
}

\begin{abstract}
We investigate the transport of granular materials by a conveyor belt via numerical simulations. We report an unusual increasing of particles horizontal velocity when they leave the belt and initiate free-fall. Using Discrete Elements Method, the mechanism underlying this phenomenon were investigated, and a study on how particle and system properties influences this effect were conducted.
\end{abstract}

\section{Introduction}

Granular materials play a fundamental role in a wide range of applications, from grain storage in the food industry to space exploration. However, even seemingly trivial questions about the behavior of these materials do not have a clear answer. [1]

A variety of different granular flows has been studied by GDR-MiDi in [2], where different flows in free fall (where the driving force is gravity) systems were characterized. Here we focus on a flow guided by a belt conveyor.

Belt conveyors are key components in any mine around the world. The velocity profile of the material being placed, transported or dropped from the belt plays an important role on the durability of mechanical components as well in the energy consumption of conveyor's drives. It is directly correlated to equipment wear and lifetime.

Via numerical simulations using molecular dynamics technique (also know as Discrete Element Method, or DEM [3]), we can evaluate and study the spatiotemporal behavior of many properties of a given granular material. We have detected a consistent increase in horizontal velocity of particles at the end of a conveyor. In this paper, we use 2D DEM simulations to demonstrate the existence of this phenomena and investigate the influence of some system parameters.

\section{Methodology}

In our studies, we use the molecular dynamics technique [4] to simulate a 2D flow of particles over a belt conveyor. The simulation model is as described in [5] and [6] adapted to incorporate objects other than particles (walls and belt), and a constant stream of particles. The contact model used for particle-object contact is the same used for particleparticle contacts.

\footnotetext{
^e-mail: abraaomt@gmail.com

${ }^{\star}$ e-mail: atman@dppg.cefetmg.br
}

As illustrated in Figure 1, the simulated base system consists of monodisperse material being transported by a conveyor-like geometry, with a $3.5 \mathrm{~m}$ long belt, an inbox for particle entrance of $1.5 \mathrm{~m}$ wide and width of $1.5 \mathrm{~m}$, with an opening for material flow of $0.5 \mathrm{~m}$. Belt velocity is constant at $3 \mathrm{~m} / \mathrm{s}$ in all simulations, this velocity is transmitted to particles without an actual movement of the geometry. An object that transmits movement without geometry displacement is a common technique used in many DEM software packages. The material being transported on this initial simulation is monodisperse (all particles have the same diameter).

For data collection we have defined 3 regions of interest, Before, During and After the belt endpoint, which is located at coordinates $(0,0)$. On a real conveyor, the belt endpoint is actually where the belt touches the pulley and particles start a ballistic movement. Results shown in this paper are based on data from particles inside these regions of interest.

On this study, we will call the velocity modulus as $V$, and it's $x$ and $y$ components as $V_{x}$ and $V_{y}$, respectively.

Particle size was chosen to balance detail level and processing time, in monodisperse simulations all particles have a radius of $0.025 \mathrm{~m}$ and between $0.023 \mathrm{~m}-0.027 \mathrm{~m}$ for polydisperse cases. Other material parameters used in this study include a normal and tangential $\operatorname{stiffness}\left(k_{n}\right.$ and $\left.k_{t}\right)$ of $10 \mathrm{E} 8$ and dumping factor $(g n)$ of 100 . Particles, walls, and belt are considered to be of the same material.

\section{Results}

The simplest evidence of the effect existence can be seen in the visualization of longitudinal component $\left(V_{x}\right)$ of the velocities of particles. On Figure 1(Right) we can observe $V_{x}$ of particles on the conveyor are equal to the belt velocity, just as expected. As particles leave the conveyor and start ballistic movement their $V_{x}$ spontaneously increases, proportional to their height on material layers, creating a 

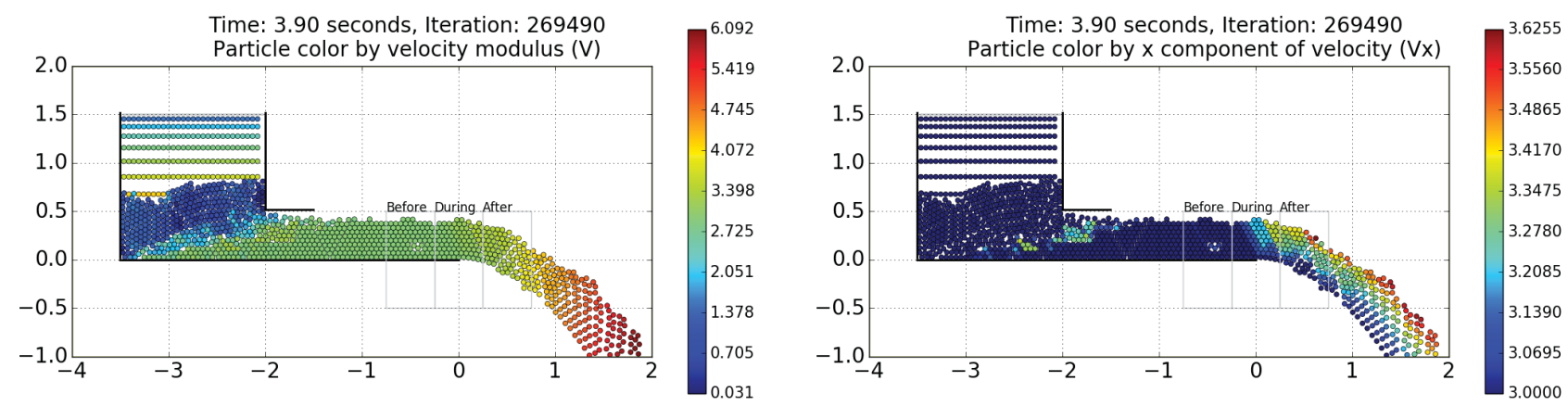

Figure 1. The simulated system: A basic conveyor with a particle inlet at the top left, a moving belt with constant velocity from left to right and three regions of interest (named "Before", "During" and "After") for data collecting. Left: Particles colored by they velocity modulus $V$, color scale automatically adjusted from minimum do maximum values in that frame. Right: Particles colored by they $V_{x}$, color scale set for range $3.0 \mathrm{~m} / \mathrm{s}$ (belt velocity) to $3.7 \mathrm{~m} / \mathrm{s}$, for detail enhancement and better visualization of the effect. Note the $V_{x}$ increasing that appears during the transition from forced flow to free fall, as well a velocity gradient from bottom to top is visible.

velocity distribution where particles next to the belt maintain their $V_{x}$ close to $3.0 \mathrm{~m} / \mathrm{s}$ and particle far from the belt increases their $V_{x}$ up to $3.8 \mathrm{~m} / \mathrm{s}(27 \%)$ in whole simulation time ( See Figure 2, $V_{x}$ after belt's end, between 6 and 7 seconds)

On Figure 2 we show the temporal evolution of $V_{x}$ in the 3 regions of interest. We show the maximum, minimum and mean values of all particles inside each region, during 7 seconds of simulation. Please note that in $t=0$ the particles start to fall from the inlet and takes about 1.0 -1.5 seconds to reach the regions of interest.

From Figure 2, $V_{x}$ before belt's end is, as expected, equal to the belt velocity with small oscillations. After belt endpoint the $V_{x}$ mean becomes higher than $3.2 \mathrm{~m} / \mathrm{s}$ and $V_{x} \max$ oscillates around $3.6 \mathrm{~m} / \mathrm{s}$.

\subsection{Parameter influence}

In our previous work [7], we have detected the existence of this phenomena in $3 \mathrm{D}$ simulations, using commercial simulation package Rocky[8]. A experiment have been designed to understand which of the three parameters height of material, friction ( between particles) and adhesion (between particles) have more influence in the effect (more influence $=$ higher $V_{x}$ ). As a result, the combination of parameters height of material and friction induced higher values of $V_{x}$. Since the company refuses to give information about the method used for they software, further investigations have to be conducted with our code.

Once demonstrated the effect existence by previous results, we can explore the influence of system parameters on it. Particle size distribution has been tested, as well material height and belt slope. Although the same overall gradient is clearly observable in all simulated systems, quantitative analyses show detailed behavior differences.

Particle size distribution: While we have used monodisperse material (all particles with the same diameter) in our base system, here the material is polydisperse, with particles diameters varying from $0.023 \mathrm{~m}-0.027 \mathrm{~m}$ chosen from a uniform distribution. All other parameters are the same as in base system.
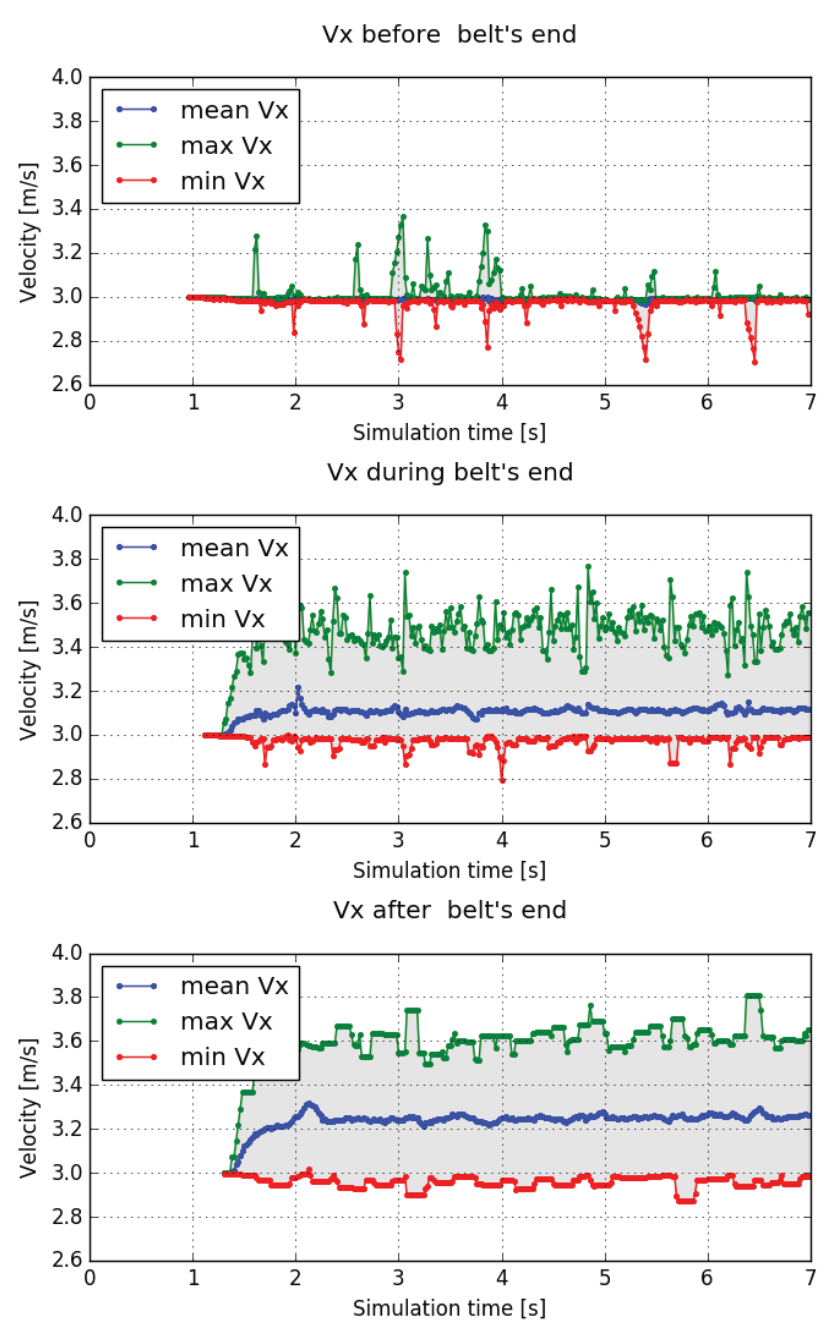

Figure 2. $V_{x} \max$, min, mean for all particles in each region of interest, in the base system (monodisperse material). It is clear the increase of maximum and mean $V_{x}$ during and after the end of the conveyor. A opposite behavior can be observed for the minimum $V_{x}$, its value became $<3.0 \mathrm{~m} / \mathrm{s}$ more frequently during the transition in the end of conveyor and always $<3.0 \mathrm{~m} / \mathrm{s}$ after the transition 

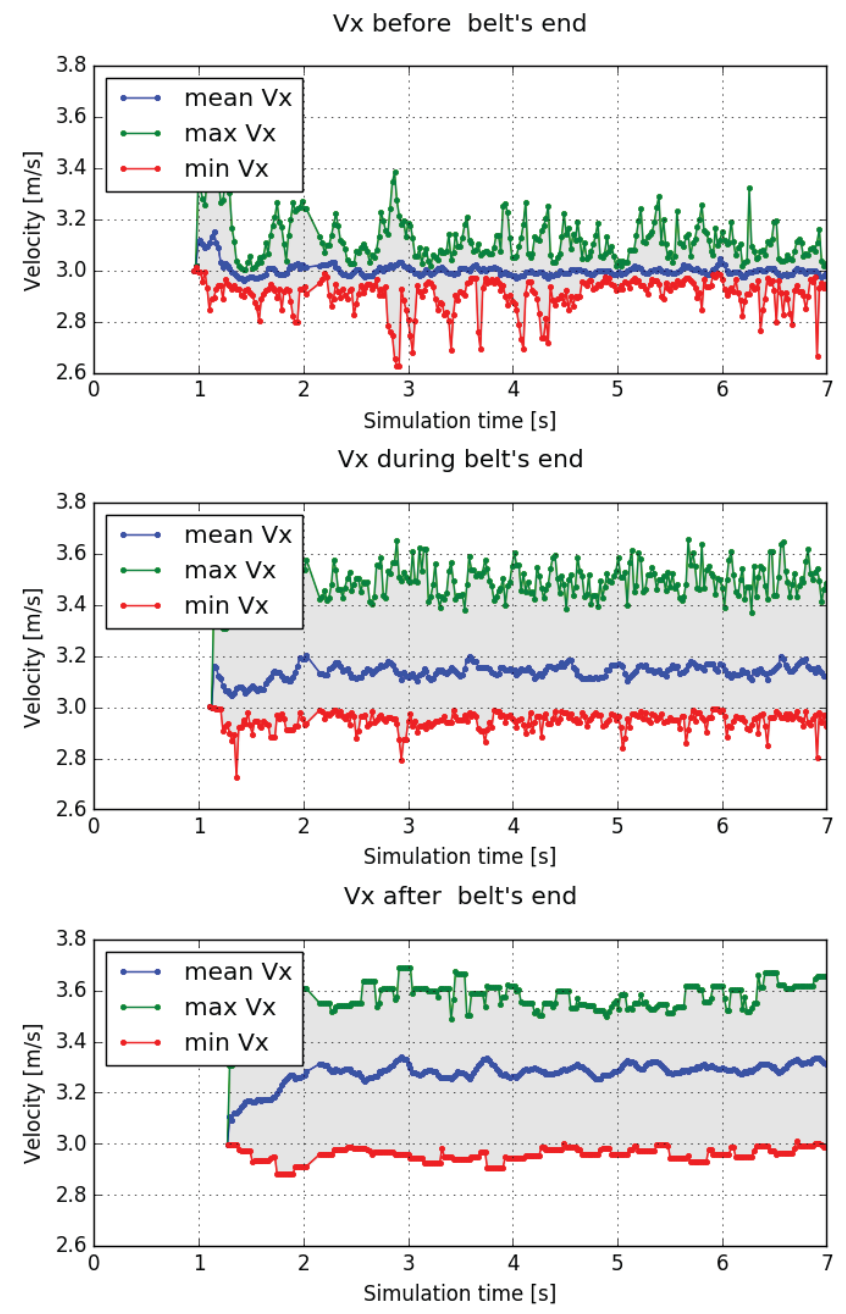

Figure 3. $V_{x} \max$, min, mean for all particles in each region of interest, in a polydisperse system. In this system, we can observe a much higher variability in $V_{x}$ before the endpoint, although the mean value stays around $3.0 \mathrm{~m} / \mathrm{s}$ as expected. Maximum $V_{x}$ after the transition is slightly less than the values obtained for the monodisperse system.

In Figure 3 is visible a much higher oscillation of maximum and minimum values on before region, and a slightly higher mean value in during and after regions. A full explanation for this observed behavior is a work in progress.

Height of the material layer: As can be inferred from the velocity gradient in Figure 1(Right), material layer height directly influences the effect. Here the test consists of tracking particles that flow in different layers and analyze the behavior of its $V_{x}$ along its trajectory.

Figure 4A shows the $x, y$ position of 4 chosen particles along their lifespan in the base system, for each of these particles Figure 4B shows its $V_{x}$ as function of $x$ coordinate. We can see that higher the material layer, higher and earlier is the increase of $V_{x}$.

Belt slope Belt conveyors are frequently designed to operate with a small slope, limited by material properties. This slope cannot be too high to avoid material rolling.
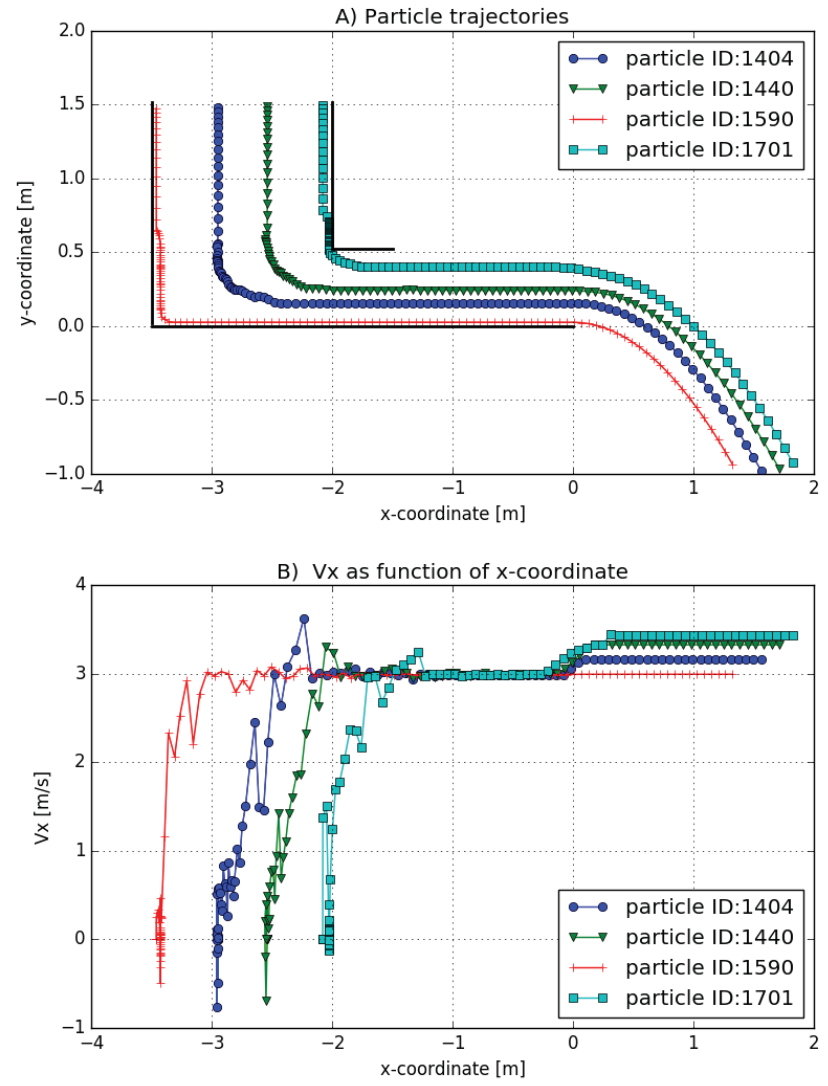

Figure 4. Influence of layer height on $V_{x}$ increase. Tracking position and $V_{x}$ of 4 particles in different layer heights, we can see the higher layers give a higher and earlier increase of $V_{x}$

Two values for belt slope $\left(+5^{\circ}\right.$ and $\left.-5^{\circ}\right)$ were tested transporting monodisperse material and compared to horizontal belt position $\left(0^{\circ}\right)$. The results in Figure 5 are obtained calculating the temporal mean of $\max V_{x}, \min V_{x}$ and mean $V_{x}$ from simulations with belt at above mentioned angles. Values before 3.0 seconds were discarded in order to consider only the flow in stationary regime. As $V_{x}$ is measured in global $X$ direction, is expected a reduction in observed $V_{x}$ in inclined systems. A particle traveling at $3.0 \mathrm{~m} / \mathrm{s}$ in a linear trajectory at $+-5^{\circ}$ has a $V_{x}$ of $3 \times \cos \left(5^{\circ}\right)=2,9885 \mathrm{~m} / \mathrm{s}$.

A positive belt angle generates an upstream flow that reduces more significantly the maximum and mean values $V_{x}$ but not the minimum $V_{x}$. In another hand, the negative slope generates a downstream flow that significantly reduces the minimum of $V_{x}$ 


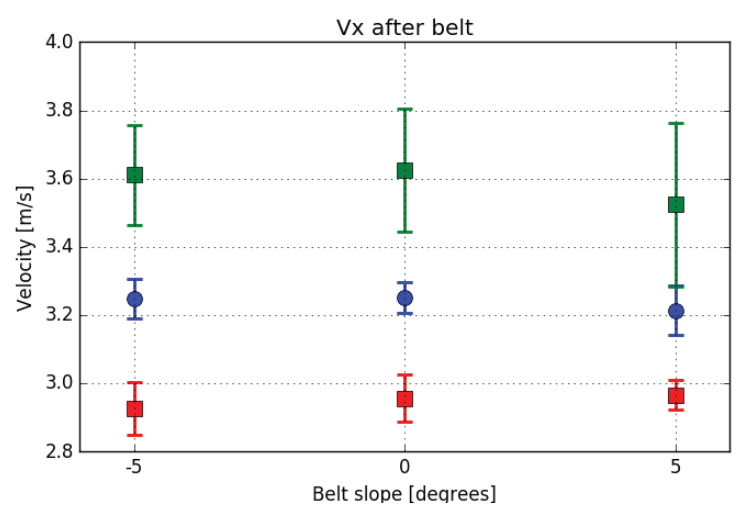

Figure 5. Results for inclining the belt. Here we show the mean values for $V_{x}$ maximum (upper squares), mean (discs), and minimum (bottom squares) averaged in time for 3 belt slope angles. Angles are measured clockwise and 0 means the base state were belt is horizontal.

\section{Conclusion}

From the moment particles being transported by a belt conveyor leave the belt, is expected that only vertical component of its velocity $\left(V_{y}\right)$ increases since gravity is the only force acting on particles.

However, we have verified via numerical simulations that the horizontal velocity of particles does increase after the belt endpoint and this effect is directly proportional to the height of material layer and influenced by particle size distribution, belt slope, friction and adhesion [7].

One possible explanation for the observed phenomena is the conservation of angular moment. Despite the absence of adhesion between particles, the natural packing and friction in the material being transported make it behave like a solid, at the transition at the end of the belt, these solid like properties are still present and the stacks of particles appear to rotate, accelerating the external ones.

Further investigation is necessary and the results presented here can guide on this path. Theoretically, measurements about this effect on real systems could be obtained by computer vision, filming the external upper and bottom layers of material on a conveyor discharge, and estimating velocities differences via optical flow technique.

\section{References}

[1] J. Duran, Sands, Powders, and Grains, Vol. 49 of Partially Ordered Systems (Springer New York, New York, NY, 2000), ISBN 978-1-4612-6790-4

[2] G.D.R. MiDi, GDR Midi, European Physical Journal E 14, 314 (2004), 0312502

[3] S. Luding, The Physics of Granular Media 3, 297 (2005)

[4] M. Allen, D. Tildesley, Computer simulation of liquids (1987)

[5] A.P.F. Atman, P. Brunet, J. Geng, G. Reydellet, G. Combe, P. Claudin, R.P. Behringer, E. Clément, Journal of Physics: Condensed Matter 17, S2391 (2005), \$411734

[6] A.P.F. Atman, P. Claudin, G. Combe, Computer Physics Communications 180, 612 (2009)

[7] A.M. Tavares, A.P.F. Atman, Estudo do aumento espontâneo na velocidade de partículas em transportadores de correia, in Proceeding Series of the Brazilian Society of Applied and Computational Mathematics, edited by M. Bernardes (SBMAC, São Carlos, SP, Brazil, 2016)

[8] ESSS, Rocky DEM, http://www.rocky-dem.com, accessed: 2016-10-15 